\title{
Efficacy of liver resection for single large hepatocellular carcinoma in Child-Pugh A cirrhosis: Analysis of nationwide cancer registry database
}

\author{
Suk Kyun HONG, Kwang-Woong LEE*, Su young HONG, Sanggyun SUH, Kwangpyo HONG, Eui Soo HAN, Jeong-Moo LEE, \\ Nam-Joon Yl, Kyung-Suk SUH \\ Department of Surgery, Seoul National University College of Medicine, Seoul, Korea
}

Introduction: Therapeutic strategies are still debated and further studies are needed on prognostic factors for patients with single large hepatocellular carcinoma (HCC). This study aimed to identify the prognostic factors of single large HCC patients with good performance status and Child-Pugh A cirrhosis using large national cancer registry database and recommend therapeutic strategy in patients with such condition.

Methods: Among 12,139 HCC patients registered at the Korean Primary Liver Cancer Registry (KPLCR) from 2008 through 2015, single large $(\geq 5 \mathrm{~cm})$ HCC patients with Eastern Cooperative Oncology Group (ECOG) performance status 0 and Child-Pugh score A were selected.

Results: 466 patients were analyzed. The 1-, 2-, 3-, and 5-year survival rates after initial treatment were $84.9 \%, 71.0 \%, 60.1 \%$, and $51.6 \%$, respectively and the 1-, 2-, 3-, and 5-year progression-free survival rates were $43.6 \%, 33.0 \%, 29.0 \%$, and $26.8 \%$, respectively. While platelet count $<100 \times 109 / \mathrm{L}(p<0.001)$, sodium level $<135 \mathrm{mmol} / \mathrm{L}(p=0.002)$, maximum tumor diameter $\geq 10 \mathrm{~cm}(p=0.001)$, and treatment other than resection (transarterial therapy vs. resection: $p<0.001$, others vs. resection: $p=0.002$ ) were significantly related to poorer overall survival, sodium $<135 \mathrm{mmol} / \mathrm{L}(p=0.015)$, maximum tumor diameter $\geq 10 \mathrm{~cm}(p<0.001)$, and treatment other than resection (transarterial therapy vs. resection: $p<0.001$, others vs. resection: $p=0.001$ ) were independently related with poorer progression-free survival.

Conclusions: Resection as initial treatment should be considered when possible even in single large HCC patient with good performance and mild cirrhosis. Physicians should pay more attention to patients with low platelet level $(<100 \times 109 / \mathrm{L})$, serum sodium level $(<135 \mathrm{mmol} / \mathrm{L})$, and maximum tumor diameter $\geq 10 \mathrm{~cm}$. 